\title{
Distributed Positioning of Autonomous Mobile Sensors with Application to Coverage Control
}

\author{
Hans-Bernd Dürr, Miloš S. Stanković and Karl Henrik Johansson
}

\begin{abstract}
We consider problems in multi-agent systems where a network of mobile sensors needs to self-organize such that some global objective function is maximized. To deal with the agents' lack of global information we approach the problem in a game-theoretic framework where agents/players are only able to access local measurements of their own local utility functions whose parameters and detailed analytical forms may be unknown. We then propose a distributed and adaptive algorithm, where each agent applies a local extremum seeking feedback adopted to its specific motion dynamics, and prove its global practical stability, implying that the agents asymptotically reach a configuration that is arbitrary close to the globally optimal one. For the stability analysis we introduce a novel methodology based on a Lie bracket trajectory approximation and combine it with a potential game approach. We apply the proposed algorithm to the sensor coverage problem and solve it in a distributed way where the agents do not need any $a$ priori knowledge about the distribution of the events to be detected and about the detection probabilities of the individual agents. The proposed scheme is illustrated through simulations.
\end{abstract}

\section{INTRODUCTION}

We focus on problems in multi-agent systems where the agents need to autonomously find positions which maximize some global objective function. These problems are typical in mobile sensor networks which consist of a collection of mobile sensing devices that coordinate their actions through wireless communication, while performing tasks such as exploration, surveillance, monitoring, target tracking, etc. The agents may have some specific motion dynamics and are autonomously moving in the plane by using only locally available information. There is no global leader and no omniscient agent that possess global information about the underlying problem.

To deal with this distributed information structure, we assign locally defined individual utility functions to each agent and interpret the problem in a game-theoretic framework. Each agent is equipped with sensors and is able to communicate with a subset of the other agents, so that it is capable of obtaining the current value of its local utility at each time instance. By formulating the problem as a potential game [8] we propose a method that guarantees convergence to a neighborhood of the global optimum, where each agent performs only local utility optimization. Since the agents can only access current values of the local utilities, whose

Hans-Bernd Dürr is with the Institute for Systems Theory and Automatic Control, University of Stuttgart, Germany; E-mail: hans-bernd.duerreist.uni-stuttgart.de

Miloš S. Stanković and Karl Henrik Johansson are with the ACCESS Linnaeus Center, School of Electrical Engineering, Royal Institute of Technology, 100-44 Stockholm, Sweden; E-mail: milsta@kth.se, kallej@kth.se parameters and detailed analytical forms may be unknown, the proposed algorithms are based on the extremum seeking scheme with periodic perturbations (see, e.g., [12], [13], [15], [16]). For the analysis of such systems we propose a novel method based on a Lie bracket system approximation (which can also be applied to general multi-variable or multiobjective ES algorithms with periodic excitations) and apply it to prove global practical asymptotic stability (cf. [9]) of the proposed schemes. This Lie bracket based approach has its origins in the controllability analysis and motion planning (see, e.g., [5], [10]). For the motion dynamics of the agents, in this paper we consider velocity actuated vehicles as well as the unicycle vehicles.

As a specific application of the proposed algorithms we consider the sensor coverage problem where a group of sensors are meant to cover a region autonomously such that the overall event detection probability is maximized. The sensors have a limited sensing and communication range, so that they are only able to measure the frequency of local events. We formulate the problem as a potential game and construct individual utility functions for each sensor such that it can be solved in a distributed and adaptive way with the proposed method.

The extremum seeking with sinusoidal perturbations by autonomous vehicles has been analyzed in [15] and [16] using the averaging theorem. The authors proposed similar schemes as in this work but provided only local stability analysis for the quadratic utilities by a single agent. In the case of noisy utility measurements these ideas were extended in [12] and [13] where the driving sinusoids admit vanishing gains so that almost sure convergence is achieved. The analysis provided in this paper, based on the Lie bracket approximation, applied even to single agent systems, provides a better qualitative description of the behavior of the original system compared to existing methods. Also, using the Lie bracket method, we are able to prove global practical stability for general nonlinear maps in a straightforward and intuitive manner. The authors of [11] extended the extremum seeking schemes to the multi-agent case when the agents are seeking for a Nash equilibrium in a stochastic environment. In [2] a similar approach but for local seeking of Nash equilibria was presented.

In [4] the sensor coverage problem, treated in this paper was introduced. The authors proposed a gradient method where each sensor moves into the direction of the steepest ascent of the utility function, thus requiring its complete analytical form. This problem was treated in a game theoretic way by the authors of [6], [7] and [17]. They proposed dif- 
ferent algorithms for a discrete action space where only one player is allowed to move at predefined time-instances and examined the convergence properties of different adaptive best or better response algorithms.

The paper is structured as follows. In Section II we recall the mathematical preliminaries that we are using throughout the paper. In Section III we prove the global practical asymptotic stability of the proposed algorithms, while in Section IV we apply them to the sensor coverage problem. In Section $\mathrm{V}$ we present simulation results.

\section{PREREQUISITES}

The set $\mathbb{R}_{+}$denotes the set of nonnegative real numbers and $\mathbb{Q}_{++}$denotes the set of positive rational numbers.

A function $f$ is said to belong to the class $C^{\infty}$ if it is smooth, or infinitely continuously differentiable (see also [3]).

A continuous function $\alpha:[0, \infty) \rightarrow[0, \infty)$ is said to belong to class $\mathcal{K}_{\infty}$ if it is strictly increasing, $\alpha(0)=0$ and $\alpha(r) \rightarrow \infty$ as $r \rightarrow \infty$.

The Jacobian of a continuously differentiable function $f$ : $\mathbb{R}^{n} \rightarrow \mathbb{R}^{m}$ with components $f(s)=\left(f_{1}(s), \ldots, f_{m}(s)\right)^{\top}$ and each $f_{i}: \mathbb{R}^{n} \rightarrow \mathbb{R}$, is denoted by

$$
\frac{\partial f(s)}{\partial s}:=\left(\begin{array}{ccc}
\frac{\partial f_{1}(s)}{\partial s_{1}} & \ldots & \frac{\partial f_{1}(s)}{\partial s_{n}} \\
\vdots & \ddots & \vdots \\
\frac{\partial f_{m}(s)}{\partial s_{1}} & \ldots & \frac{\partial f_{m}(s)}{\partial s_{n}}
\end{array}\right) .
$$

The gradient of a continuously differentiable function $Q: \mathbb{R}^{n} \rightarrow \mathbb{R}$ with respect to $s$ is denoted by $\nabla_{s} Q(s):=\left(\frac{\partial Q(s)}{\partial s_{1}}, \ldots, \frac{\partial Q(s)}{\partial s_{n}}\right)^{\top}$ and $\left(\nabla_{s} Q\right)^{2}$ stands for $\nabla_{s} Q(s)^{\top} \nabla_{s} Q(s)$.

The norm $\|\cdot\| C[0, T]$ denotes $\|y\|_{C[0, T]}=$ $\max _{t \in[0, T]}|y(t)|$.

The Lie bracket (cf. [10]) of two vector fields $f$ and $g$ is defined as $[f, g]=\frac{\partial g}{\partial s} f-\frac{\partial f}{\partial s} g$.

We now show how an input-affine system can be approximated by an extended system consisting of vector-fields calculated from Lie brackets. Consider the following system

$$
\dot{x}=\sum_{i=1}^{m} b_{i}(x) u_{i}^{\epsilon}, \quad x \in \mathbb{R}^{n}, \quad b_{i}(x) \in C^{\infty}: \mathbb{R}^{n} \rightarrow \mathbb{R}^{n}
$$

with inputs $u_{i}^{\epsilon}=\bar{u}_{i}(t)+\frac{1}{\sqrt{\epsilon}} \tilde{u}_{i}(t, \theta), \epsilon>0$, where $\tilde{u}_{i}$ is $2 \pi$-periodic in $\theta=t / \epsilon$, and has zero average, i.e., $\int_{0}^{2 \pi} \tilde{u}_{i}(t, \theta) d \theta=0$.

Consider also the system

$$
\dot{z}=\sum_{i=1}^{m} b_{i}(z) \bar{u}_{i}+\frac{1}{2 \pi} \sum_{i<j}\left[b_{i}, b_{j}\right] \nu_{i, j}, \quad z(0)=x(0),
$$

where

$$
\nu_{i, j}=\int_{0}^{2 \pi} \int_{0}^{\theta} \tilde{u}_{i}(t, \tau) \tilde{u}_{j}(t, \theta) d \tau d \theta .
$$

The following lemma states the connection between these two systems in terms of the difference in their trajectories, by giving a bound that tends to zero as $\epsilon$ tends to zero.

Lemma 1 (Thm. 2.1 in [5] p. 68): For sufficiently small $\epsilon>0$, the trajectory of the system (1), is bounded by the solution of the system (2) in the sense that

$$
\|x-z\|_{C[0,2 \pi]} \leq \Delta_{\epsilon}
$$

where $\Delta_{\epsilon}$ is a parameter that tend to zero as $\epsilon \rightarrow 0$.

From Lemma 1 it is easy to show that the trajectory of system (1) converge uniformly on any compact time interval to the trajectory of (2) as $\epsilon \rightarrow 0$. Under these conditions the following holds:

Lemma 2 (cf. [9]): If the origin is a globally uniformly asymptotically stable equilibrium point of system (2), then for sufficiently small $\epsilon>0$ the origin of system (1) is practically globally uniformly asymptotically stable.

This lemma is a special case of the original statement in [9] and can easily be proven using the Gronwall-Bellman Lemma. By performing a change of variables the result can be extended to any point in the state space.

Unlike the asymptotic stability in the sense of Lyapunov, where trajectories converge to the origin as time goes to infinity, the notion of practical stability of time-varying systems deals with the trajectory convergence to a region containing the origin, which can be made arbitrary small by tuning certain system parameters. The existence of such parameters makes the notion of practical stability different to the convergence to a limit cycle.

\section{MAIN RESULTS}

In this section we propose two distributed multi-agent extremum seeking algorithms based on a game-theoretic methodology. The first one is applied to the single integrator model of the agents' motion dynamics, whereas the second one is applied to the unicycle model.

The position vector of each agent, is denoted by $s_{i}=$ $\left(s_{i x}, s_{i y}\right)^{\top} \in \mathbb{R}^{2}$.

Let us assume that the positions of the agents can be interpreted as their actions in a potential game $\Delta=\langle V, A, U\rangle$, where $V=(1, \ldots, N)$ is the set of players/agents, $A:=$ $\prod_{i=1}^{N} \mathbb{R}^{2}$ is the action set and $U=\left\{v_{i}(s): A \rightarrow \mathbb{R}, i \in V\right\}$ with $s=\left[s_{1}^{T}, \ldots, s_{N}^{T}\right]^{T}$, is a set of utility functions. The potential function $Q(s): A \rightarrow \mathbb{R}$ is continuously differentiable, strictly concave and admits a single maximum at $s^{*}$ which, therefore coincides with a unique Nash equilibrium in purestrategies. Note that we use the definition for potential games in [8], where the function $Q(s)$ is a potential function for the game $\Delta$ if and only if

$$
\nabla_{s_{i}} Q(s)=\nabla_{s_{i}} v_{i}(s), \quad \forall i \in V .
$$

\section{A. Single Integrator Motion Dynamics}

Let us consider the state-space of the extremum seeking feedback given in Fig. 1. We introduce the following assumptions on the parameters of the algorithm and of the Game $\Delta$ : 


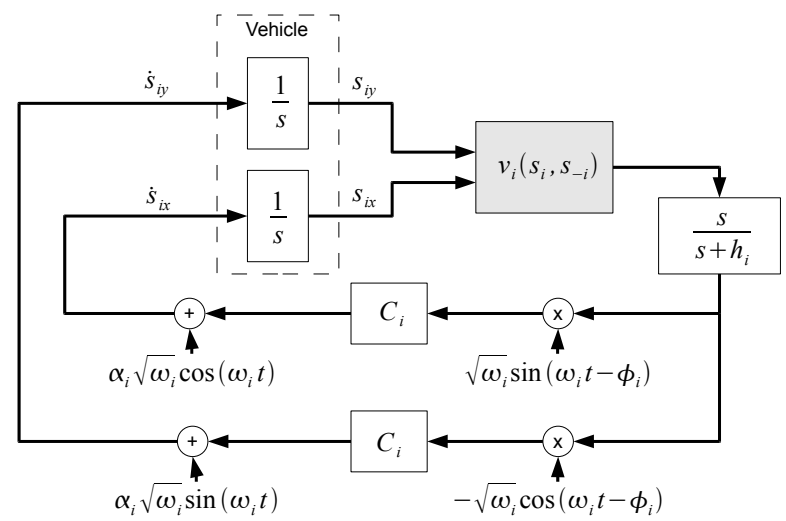

Fig. 1: Single Agent Equipped with the Extremum Seeking Feedback

A.1 $\omega_{i}=a_{i} \omega$ and $a_{i} \neq a_{j}, \forall i \neq j, a_{i} \in \mathbb{Q}_{++}, \omega \in(0, \infty)$, A. $2-\frac{\pi}{2}<\phi_{i}<\frac{\pi}{2}, \forall i \in V$,

A. $3 h_{i}>0, \alpha_{i}>0, c_{i}>0, \forall i \in V$,

A.4 $v_{i}(s)$ is smooth, $\forall i \in V$.

The following theorem deals with the practical stability of the proposed scheme.

Theorem 1: Consider the system of $N$ agents equipped with the extremum seeking feedback in Fig. 1. Under the Assumptions A.1-A.4 and for sufficiently large $\omega$, the Nash equilibrium $s^{*}$ of the Game $\Delta$ is practically globally uniformly asymptotically stable.

Proof: We write $v_{i}$ instead of $v_{i}(s)$. The idea is to bring the system into the form (1) and derive the approximative system as in (2) that can easily be analyzed with standard Lyapunov techniques. By using the identities $\sin (x-y)=\sin (x) \cos (y)-\cos (x) \sin (y)$ and $\cos (x-y)=$ $\cos (x) \cos (y)+\sin (x) \sin (y)$, the system equations for each agent $i$ have the following structure

$$
\begin{aligned}
\dot{s}_{i x}= & c_{i}\left(v_{i}-e_{i} h_{i}\right) \sqrt{\omega_{i}} \sin \left(a_{i} \omega t\right) \cos \left(\phi_{i}\right) \\
& -c_{i}\left(v_{i}-e_{i} h_{i}\right) \sqrt{\omega_{i}} \cos \left(a_{i} \omega t\right) \sin \left(\phi_{i}\right) \\
& +\alpha_{i} \sqrt{\omega_{i}} \cos \left(a_{i} \omega t\right) \\
\dot{s}_{i y}= & -c_{i}\left(v_{i}-e_{i} h_{i}\right) \sqrt{\omega_{i}} \cos \left(a_{i} \omega t\right) \cos \left(\phi_{i}\right) \\
& -c_{i}\left(v_{i}-e_{i} h_{i}\right) \sqrt{\omega_{i}} \sin \left(a_{i} \omega t\right) \sin \left(\phi_{i}\right) \\
& +\alpha_{i} \sqrt{\omega_{i}} \sin \left(a_{i} \omega t\right) \\
\dot{e}_{i}= & -e_{i} h_{i}+v_{i}
\end{aligned}
$$

where the filter of agent $i$ is represented by the equivalent state-space model whose internal state is denoted by $e_{i}$ and whose output is $y_{i}=-e_{i} h_{i}+v_{i}$. The position vectors and the states of the filter of each agent are stacked in a vector $(s, e)^{\top}:=\left(s_{1 x}, s_{1 y}, e_{1}, \ldots, s_{N x}, s_{N y}, e_{N}\right)^{\top}$ and all driving sinusoids with the same frequency are collected together to obtain

$$
\left(\begin{array}{c}
\dot{s} \\
\dot{e}
\end{array}\right)=\sum_{i=1}^{N} b_{i a} \underbrace{\sqrt{\omega_{i}} \sin \left(a_{i} \omega t\right)}_{u_{i a}}+b_{i b} \underbrace{\sqrt{\omega_{i}} \cos \left(a_{i} \omega t\right)}_{u_{i b}}+b_{i e}
$$

where $b_{i a}$ and $b_{i b}$ are the vector fields with entries only at the components for the respective agent and all other components are zero. The vector field $b_{i e}$ consists of entries $-e_{i} h_{i}+v_{i}$ for the filter of each agent. These definitions directly follow from (6).

By Assumption A.1 all $a_{i}$ can be written as $a_{i}=p_{i} / q_{i}$ with $p_{i}, q_{i} \in \mathbb{N}$.

Choose $q:=\prod_{j} q_{j}, \epsilon:=q / \omega$ and define $\tilde{\alpha}_{i}:=\alpha_{i} \sqrt{p_{i} \prod_{j \neq i} q_{j}}, \quad \tilde{c}_{i}:=c_{i} \sqrt{p_{i} \prod_{j \neq i} q_{j}}, \quad \tilde{u}_{i a}=$ $\sqrt{\omega / q} \sin \left(a_{i} \omega t\right)$ and $\tilde{u}_{i b}=\sqrt{\omega / q} \cos \left(a_{i} \omega t\right)$. Equation (7) can be written using vector fields $\tilde{b}_{i a}$ and $\tilde{b}_{i b}$ obtained from $b_{i a}$ and $b_{i b}$ using $\tilde{\alpha}_{i}$ and $\tilde{c}_{i}$ instead of $\alpha_{i}$ and $c_{i}$. The corresponding inputs are $\tilde{u}_{i a}=1 / \sqrt{\epsilon} \sin \left(p_{i} \prod_{j \neq i} q_{j} t / \epsilon\right)$ and $\tilde{u}_{i b}=1 / \sqrt{\epsilon} \cos \left(p_{i} \prod_{j \neq i} q_{j} t / \epsilon\right)$ where by assumption $p_{i} \prod_{j \neq i} q_{j} \in \mathbb{N}$.

The drift influences only the state $e_{i}$ to which a virtual input $u_{i 0}=1$ is associated. All $\tilde{u}_{i a}$ 's and $\tilde{u}_{i b}$ 's are periodic in $2 \pi$ and their averages are zero.

$$
\int_{0}^{2 \pi} \sin \left(p_{i} \prod_{j \neq i} q_{j} \theta\right) d \theta=\int_{0}^{2 \pi} \cos \left(p_{i} \prod_{j \neq i} q_{j} \theta\right) d \theta=0 .
$$

Therefore all assumptions of Lemma 1 are fulfilled, and can be applied. The approximate system is

$$
\left(\begin{array}{c}
\dot{\bar{s}} \\
\dot{\bar{e}}
\end{array}\right)=\frac{1}{2 \pi} \sum_{i<j}\left[\tilde{b}_{i}, \tilde{b}_{j}\right] \nu_{i, j}+\sum_{i} b_{i e}
$$

with $\nu_{i, j}=\int_{0}^{2 \pi} \int_{0}^{\theta} u_{i}(\tau) u_{j}(\theta) d \tau d \theta$. The summation is done over all $i \in\{1 a, 1 b, 2 a, 2 b, \ldots\}$. An important fact now is that $\nu_{i, j}=0$ for the coupling Lie brackets of agents with different frequencies. Note that the Lie bracket applied to one vector field equals zero $\left(\left[\tilde{b}_{i a}, \tilde{b}_{i a}\right]=0\right)$. As in Eq. (2) the $\nu_{i, j}$ are only to be calculated for $i<j$. After a lengthly calculation we obtain for the approximate system of agent $i$

$$
\begin{aligned}
\dot{\bar{s}}_{i x}= & \frac{1}{2}\left(c_{i} \alpha_{i} \nabla_{\bar{s}_{i x}} v_{i}(\bar{s}) \cos \left(\phi_{i}\right)+c_{i} \alpha_{i} \nabla_{\bar{s}_{i y}} v_{i}(\bar{s}) \sin \left(\phi_{i}\right)\right. \\
& \left.-c_{i}^{2} \nabla_{\bar{s}_{i y}} v_{i}(\bar{s})\left(v_{i}(\bar{s})-\bar{e}_{i} h\right)\right) \\
\dot{\bar{s}}_{i y}= & \frac{1}{2}\left(c_{i} \alpha_{i} \nabla_{\bar{s}_{i y}} v_{i}(\bar{s}) \cos \left(\phi_{i}\right)-c_{i} \alpha_{i} \nabla_{\bar{s}_{i x}} v_{i}(\bar{s}) \sin \left(\phi_{i}\right)\right. \\
& \left.+c_{i}^{2} \nabla_{\bar{s}_{i x}} v_{i}(\bar{s})\left(v_{i}(\bar{s})-\bar{e}_{i} h\right)\right) \\
\dot{\bar{e}}_{i}= & -\bar{e}_{i} h_{i}+v_{i}(\bar{s}) .
\end{aligned}
$$

Note, that the approximate system for each agent is only coupled in the individual utility functions $v_{i}$. Therefore, according to Lemma 1, we can conclude that the trajectories of the original system are bounded by the trajectories of the above system in the sense that $\left\|(s, e)^{\top}-(\bar{s}, \bar{e})^{\top}\right\|_{C[0,2 \pi]} \leq \Delta_{\epsilon}$.

The position vectors of each agent can now be treated separately from the filter states. Let's consider the reduced system consisting only of the position vectors $s=$ $\left[s_{1 x}, s_{1 y}, \ldots, s_{N x}, s_{N y}\right]$.

Using the potential function $W=-Q(\bar{s})+Q\left(s^{*}\right)$ as a Lyapunov function and performing the change of variables $\tilde{s}:=\bar{s}-s^{*}$, one obtains for the derivative along the trajectories of the approximative system

$$
\begin{aligned}
\dot{W}= & -\nabla_{\tilde{s}_{1 x}} Q\left(\tilde{s}+s^{*}\right) \dot{\tilde{s}}_{1 x}-\nabla_{\tilde{s}_{1 y}} Q\left(\tilde{s}+s^{*}\right) \dot{\tilde{s}}_{1 y} \\
& -\ldots \\
& -\nabla_{\tilde{s}_{N x}} Q\left(\tilde{s}+s^{*}\right) \dot{\tilde{s}}_{1 x}-\nabla_{\tilde{s}_{N y}} Q\left(\tilde{s}+s^{*}\right) \dot{\tilde{s}}_{N y} .
\end{aligned}
$$




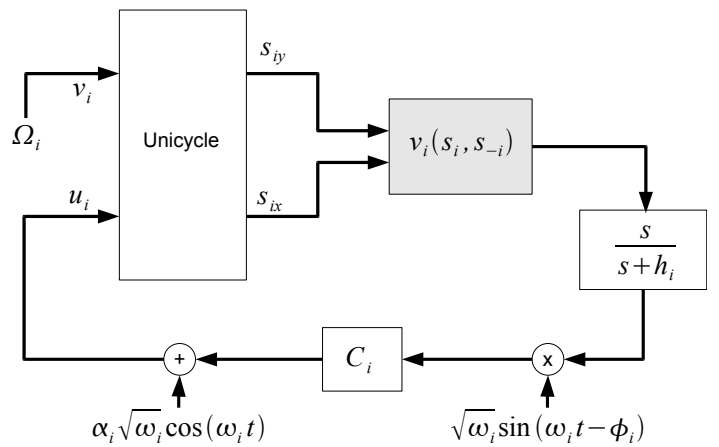

Fig. 2: Unicycle Agent Equipped with the Extremum Seeking Feedback

As this is a potential game, the individual utility functions fulfill the identity $\nabla_{s_{i}} Q=\nabla_{s_{i}} v_{i}$. This yields

$$
\begin{aligned}
\dot{W}= & -\frac{c_{1} \alpha_{1}}{2}\left(\nabla_{\tilde{s}_{1 x}} Q\left(\tilde{s}+s^{*}\right)\right)^{2} \cos \left(\phi_{1}\right) \\
& -\ldots \\
& -\frac{c_{N} \alpha_{N}}{2}\left(\nabla_{\tilde{s}_{N y}} Q\left(\tilde{s}+s^{*}\right)\right)^{2} \cos \left(\phi_{N}\right) \\
& <0 \quad \forall \tilde{s} \neq 0,
\end{aligned}
$$

since, by Assumption A.2, $\cos \left(\phi_{i}\right)$ is always positive. Therefore, the maximum $s^{*}$ is globally uniformly asymptotically stable for the first part of the approximate system (10). Consider now

$$
\dot{\bar{e}}_{i}=-\bar{e}_{i} h_{i}+v_{i} .
$$

Obviously, all $\bar{e}_{i}$ 's are decoupled and input-to-state stable with respect to $v_{i}(\bar{s})$ (which are bounded and smooth). Therefore, $\bar{e}_{i} \rightarrow v_{i}\left(s^{*}\right) / h_{i}$ for $t \rightarrow \infty$, having in mind that $s \rightarrow s^{*}$. We conclude by Lemma 2 that $s^{*}$ is practically globally uniformly asymptotically stable for the original system.

\section{B. Unicycle Motion Dynamics}

Let us consider the unicycle model for each agent given by the equations

$$
\begin{aligned}
\dot{s}_{i x} & =u_{i} \cos \left(\theta_{i}\right) \\
\dot{s}_{i y} & =u_{i} \sin \left(\theta_{i}\right) \\
\dot{\theta}_{i} & =v_{i} .
\end{aligned}
$$

The extremum seeking feedback controls only the forward velocity of the vehicle, whereas the angular velocity is constant, so that the inputs to each vehicle are $u_{i}=\left(c_{i}\left(v_{i}-e_{i} h_{i}\right) \sqrt{\omega_{i}} \sin \left(\omega_{i} t-\phi_{i}\right)+\alpha_{i} \sqrt{\omega_{i}} \cos \left(\omega_{i} t\right)\right)$ and $v_{i}=\Omega_{i}$. We make the following assumptions on the parameters of the scheme:

B.1 $\omega_{i}=a_{i} \omega$ and $a_{i} \neq a_{j}, \quad \forall i \neq j, a_{i} \in \mathbb{Q}_{++}, \omega \in(0, \infty)$, B. $2-\frac{\pi}{2}<\phi_{i}<\frac{\pi}{2}, \forall i \in V$,

B.3 $h_{i}>0, \alpha_{i}>0, c_{i}>0, \Omega_{i} \neq 0, \forall i \in V$,

B.4 $v_{i}(s)$ is smooth, $\forall i \in V$.

Theorem 2: Consider the system of $N$ agents equipped with the extremum seeking feedback in Fig. 2. Under the Assumptions B.1-B.4 and for sufficiently large $\omega$, the Nash equilibrium $s^{*}$ of the Game $\Delta$ is practically globally uniformly asymptotically stable.

Proof: We first plug the given inputs into the system equations of the unicycle model. By using the identitiy $\sin (x-y)=\sin (x) \cos (y)-\cos (x) \sin (y)$ we obtain

$$
\begin{aligned}
\dot{s}_{i x}= & \left(c_{i}\left(v_{i}-e_{i} h_{i}\right) \sqrt{\omega_{i}} \sin \left(a_{i} \omega t\right) \cos \left(\phi_{i}\right)\right. \\
& -c_{i}\left(v_{i}-e_{i} h_{i}\right) \sqrt{\omega_{i}} \cos \left(a_{i} \omega t\right) \sin \left(\phi_{i}\right) \\
& \left.+\alpha_{i} \sqrt{\omega_{i}} \cos \left(a_{i} \omega t\right)\right) \cos \left(\theta_{i}\right) \\
\dot{s}_{i y}= & \left(c_{i}\left(v_{i}-e_{i} h_{i}\right) \sqrt{\omega_{i}} \sin \left(a_{i} \omega t\right) \cos \left(\phi_{i}\right)\right. \\
& -c_{i}\left(v_{i}-e_{i} h_{i}\right) \sqrt{\omega_{i}} \cos \left(a_{i} \omega t\right) \sin \left(\phi_{i}\right) \\
& \left.+\alpha_{i} \sqrt{\omega_{i}} \cos \left(a_{i} \omega t\right)\right) \sin \left(\theta_{i}\right) \\
\dot{\theta}_{i}= & \Omega_{i} \\
\dot{e}_{i}= & -e_{i} h_{i}+v_{i} .
\end{aligned}
$$

The rest of the proof is similar as for the single-integrator. By calculating the approximative system using Lie brackets and by applying standard Lyapunov techniques to prove its asymptotic stability, we conclude by Lemma 2 that $s^{*}$ is practically globally uniformly asymptotically stable.

\section{Remarks}

The Assumptions A.1 and B.1 make sure that the motions of neighboring agents are decoupled. Simulations have shown that irrational multiples of the same frequency lead to the same results, whereas the same frequencies for all agents leads to divergence.

The presented results can be extended to agents with double-integrator dynamics [1]. By adding low-pass compensators and some additional assumptions, the practical stability can be proved in the same way.

The choice of the individual utility functions $v_{i}(s)$ will depend on the particular problem setup. In most of the practical applications some form of communication among the agents is needed to obtain local measurements. In such cases, the utility functions should be designed such that the agents can obtain local measurements by only communicating with the neighboring agents. One example of such a design will be treated in the following section.

\section{SENSOR COVERAGE AS POTENTIAL GAME}

We are going to apply the proposed algorithms to a sensor coverage problem [4]. Given a mission space $\Omega \subseteq \mathbb{R}^{2}$ where an event density function $R(x): \Omega \rightarrow \mathbb{R}_{+}$is defined. $N$ agents are placed in the mission space, whereas the position of every agent $i$ is denoted by $s_{i} \in \Omega$. The detection probability $p_{i}\left(x, s_{i}\right): \Omega \times \Omega \rightarrow[0,1]$ is a function, decaying with $\left\|x-s_{i}\right\|$, giving a measure of the probability that agent $i$ detects an event at position $x$.

The overall objective of the multi-agent sensor system can be written as the integral expression

$$
F(s)=\int_{\Omega} R(x)\left[1-\prod_{i=1}^{N}\left(1-p_{i}\left(x, s_{i}\right)\right)\right] d x .
$$


It characterizes the overall events detection frequency in terms of the positions of all the agents, since the term $\left[1-\prod_{i=1}^{N}\left(1-p_{i}\left(x, s_{i}\right)\right)\right]$ denotes the probability that an event at the position $x$ is detected by at least one agent. In terms of discrete events, the function $F(s)$ can be interpreted as being proportional to the number of events detected by at least one agent in some large enough time period. The following theorem defines the coverage problem as a potential game.

Theorem 3 (Sensor Coverage Game): The Game $\Gamma=$ $\langle V, A, U\rangle$ where $V:=\{1, \ldots, N\}$ is the player set, $A=$ $\prod_{i=1}^{N} A_{i}$ the mission space, $U=\left\{u_{i}(s): A \rightarrow \mathbb{R}, i \in V\right\}$ with

$$
u_{i}(s)=\int_{\Omega} R(x) p_{i}\left(x, s_{i}\right)\left[\prod_{\substack{j=1 \\ j \neq i}}^{N}\left(1-p_{j}\left(x, s_{j}\right)\right)\right] d x
$$

where $R(x)$ and $p_{i}\left(x, s_{i}\right)$ are continuously differentiable functions on the domain $\Omega$, is a potential game with potential function in Eq. (15).

Proof: By differentiating the potential function $F(s)$ with respect to $s_{i}$ one can easily verify that this is equal to the derivative of $u_{i}$ with respect to $s_{i}$.

$$
\nabla_{s_{i}} F(s)=\nabla_{s_{i}} u_{i}(s), \quad \forall i \in V .
$$

The fact that $R(x)$ and $p_{i}\left(x, s_{i}\right)$ are continuously differentiable allows to exchange the differentiation and integration.

The individual utility functions $u_{i}(s)$ were constructed using the Wonderful Life Utility introduced by D. Wolpert in [14] and are the continuous version of the ones proposed by the authors in [6]. It measures the marginal contribution of an agent w.r.t. the potential function. The utility functions also have a physical meaning and define the amount of detected events only by agent $i$. Therefore, the individual utility function can be measured by counting the events that were only detected by agent $i$ and by none of the others. Assuming that the detection range of each agent (defined by $\left.p_{i}\left(x, s_{i}\right)\right)$ is restricted to a small region around the agent, it is obvious that the agents only need the information from the neighbors.

\section{A. Optimal Positioning in the Sensor Coverage Game}

In order to apply the proposed algorithms to the sensor coverage game, suitable functions $R(x)$ and $p_{i}\left(x, s_{i}\right)$ have to be found. We choose the mission space to be $\Omega=\mathbb{R}^{2}$ and we make the following assumptions

C.1 $R(x) \in C^{\infty}$,

C.2 $p_{i}\left(x, s_{i}\right) \in C^{\infty}$ with compact support, $p\left(s_{i}, s_{i}\right) \neq 0$

C. $3 \int_{\mathbb{R}^{2}} R(x) p_{i}\left(x, s_{i}\right) d x \rightarrow 0$ with $\left\|s_{i}\right\| \rightarrow \infty$.

C.4 Every local maximum of $F(s)$ is an isolated point in $\mathbb{R}^{2 N}$.

Due to Assumptions C.2 and C.3 the potential function $F(s)$ admits at least one local maximum. The value of $F(s)$ would decrease with $s_{i} \rightarrow \infty$ because all $p_{i}\left(x, s_{i}\right)$ have a compact support with center at $s_{i}$. The agents search to agglomerate close to a maximum of $R(x)$ as the probability of detecting an event is maximal. Therefore, no local maximum of $F(s)$ is at $s_{i} \rightarrow \infty$.

Although the potential function is in general not strictly concave, it will still be possible to converge to a local maximum depending on the initial condition for the Lie bracket approximation. This result is similar to the notion of basin of attraction of a dynamical system with multiple local equilibria. For sufficiently large $\omega$, the original extremum seeking will be close enough to the trajectories of the Lie bracket system.

By the same reasoning as in Theorem 1, the next theorem follows:

Corollary 1: Let the Assumptions A.1 - A.4 and C.1 C. 4 be satisfied. If the agents are equipped with the extremum seeking feedback in Fig. 1, with $v_{i}(s)=u_{i}(s)$ as in equation (16), then, for sufficiently large $\omega$, every local maximum of $F(s)$ is practically uniformly asymptotically stable.

The same reasoning can be made from Theorem 2 for the unicycle model.

Corollary 2: Let the assumptions B. 1 - B.4 and C. 1 - C. 4 be satisfied. If the agents are equipped with the extremum seeking feedback in Fig. 2, with $v_{i}(s)=u_{i}(s)$ as in equation (16), then, for sufficiently large $\omega$, every local maximum of $F(s)$ is practically uniformly asymptotically stable.

Practical uniform asymptotic stability can be defined similarly as global practical uniform asymptotic stability (cf. [9]) but for a restricted set of initial conditions.

We assumed that the local maxima of the potential function $F(s)$ are isolated points. In the sensor coverage problem this is not always the case, as symmetry leads to a manifold of equal values of $F(s)$. In this case, by using the proposed schemes, convergence to a manifold $S^{*}$ is achieved in the above practical sense, where $S^{*} \subseteq \mathbb{R}^{2}$, such that for all $s \in S^{*}$ the potential function $F(s)$ takes the same local maximal value.

It is obvious that, in our case, all equilibria of the potential function are steady states for the approximative Lie bracket system. Therefore, local minima and saddle-points have to be excluded as initial conditions. Nevertheless, because of the periodic excitation in the extremum seeking, the agents will always diverge from all unstable extremum points of the potential function, and converge to a local maximum using the same reasoning as in the proofs of Theorem 1 and 2 .

To obtain the current values of the utility functions (16) the agents can rely only on locally available information having in mind that the functions $p_{i}\left(x, s_{i}\right)$ have compact support so that only the neighboring agents, which are currently in this small region around the agent, can affect their local utilities. Furthermore, it is important to observe that our algorithm is adaptive (based only on the measurements of the local utilities), so that the agents do not need any a priori knowledge about the distribution of the events to be detected $R(x)$ and about the detection probability functions $p_{i}\left(x, s_{i}\right)$ of the individual agents. 


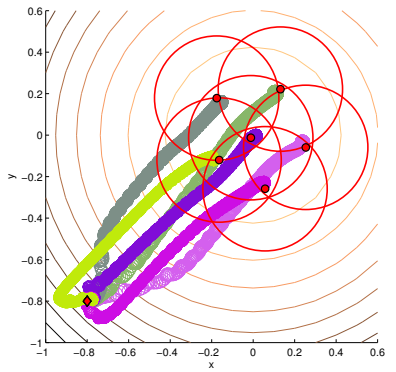

(a) Trajectories of Agents

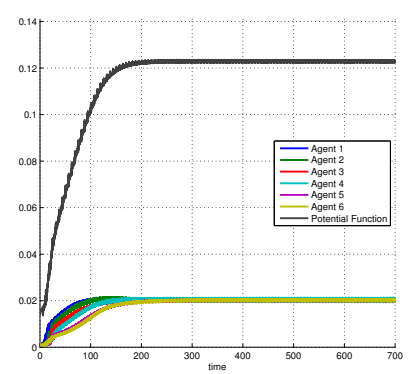

(b) Utility Functions
Fig. 3: Coverage Control Example

\section{SIMULATION RESULTS}

Consider a Gaussian distribution for $R(x)$ with mean $\mu$ and variance $\Sigma$ and

$p_{i}\left(x, s_{i}\right)= \begin{cases}p_{0 i} e^{-\frac{1}{r_{\max _{i}}^{2}-\left\|x-s_{i}\right\|_{2}^{2}}+\frac{1}{r_{\max _{i}}^{2}}} & \left\|x-s_{i}\right\| \leq r_{\max _{i}} \\ 0 & \text { else }\end{cases}$

where $\|y\|_{2}$ denotes the euclidian norm of the vector $y$. The parameters $p_{0 i}$ define the detection probability at $x=s_{i}$ and $r_{\max _{i}}$ defines the maximal detection radius of agent $i$.

We present the results for the single-integrator models. The simulations are done with $N=6$ agents. For the mean of the Gaussian distribution we choose $\mu=(0,0)^{\top}$, and for the covariance we choose the identity matrix. For the parameter of the detection probabilities of the agents, we take $p_{0 i}=1$ and $r_{\max _{i}}=0.3$. The individual frequencies for the agents are $\omega_{i}=\{10,11,12,13,14,15\}$ for each agent respectively. The parameter $\phi_{i}$ for the phase shift of the sinusoids is always $\phi_{i}=0$, whereas $\alpha_{i}=0.1, h_{i}=1$ and $c_{i}=10$, for all $i$.

The resulting trajectories of the agents are shown in Fig. 3a. The detection regions of all the agents are drawn as circles and the iso-levels where $R(x)=$ const. are drawn in the background. In Fig. 3b the evolution of the individual utility functions as well as of the potential function can be seen. The value of the potential function is monotonically increasing over time although this cannot be directly concluded from the values of the individual utility functions.

\section{CONCLUSION}

We have proved practical stability of a Nash equilibrium in a potential game in which the agents are using extremum seeking as a local optimization algorithm. The agents are able to converge to an optimum by using only the current values of their individual utility functions. We analyzed the proposed systems using the Lie bracket trajectory approximation which opens up a novel and intuitive view to the general extremum seeking schemes based on periodic excitations. We applied the proposed method to the sensor coverage problem where the individual utility functions were constructed such that the problem can be formulated as a potential game. All local Nash equilibria are practically asymptotically stable and therefore all sensors equipped with the proposed extremum seeking schemes converge arbitrary close to one of the local Nash equilibria. Due to the nature of the proposed algorithm, the agents only need to use locally available information, without any a priori knowledge about the parameters and analytical forms of the utility functions.

As a possible future research, the Lie bracket interpretation of the extremum seeking algorithms opens up possibility to include additional collision avoidance feedback and to prove convergence even in the presence of obstacles.

\section{ACKNOWLEDGMENTS}

We would like to thank Shankar Sastry and Christian Ebenbauer for fruitful discussions. We acknowledge the Deutschen Akademischen Austauschdienst (DAAD) for the financial support. This work was also supported by the Knut and Alice Wallenberg Foundation, the Swedish Research Council and the Swedish Strategic Research Foundation.

\section{REFERENCES}

[1] H.-B. Dürr. Distributed positioning of autonomous mobile sensors with application to the coverage problem. Master's thesis, Royal Institute of Technology, Stockholm Sweden, 2010.

[2] P. Frihauf, M. Krstic, and T. Basar. Nash equilibrium seeking for dynamic systems with non-quadratic payoffs. In The 14th International Symposium on Dynamic Games and Applications, 2010.

[3] H. K. Khalil. Nonlinear systems. Prentice Hall, Upper Saddle River, N.J., 3rd ed edition, 2002.

[4] W. Li and C. Cassandras. Distributed cooperative coverage control of sensor networks. In 44th IEEE Conference on Decision and Control and European Control Conference. CDC-ECC '05., pages 2542 2547, Dec. 2005.

[5] Z. Li and J. F. Canny, editors. Nonholonomic Motion Planning. Kluwer Academic Publishers, Norwell, MA, USA, 1992.

[6] J. Marden, G. Arslan, and J. Shamma. Cooperative control and potential games. Systems, Man, and Cybernetics, Part B: Cybernetics, IEEE Transactions on, pages 1393 - 1407, Dec. 2009.

[7] J. Marden, H. Young, G. Arslan, and J. Shamma. Payoff based dynamics for multi-player weakly acyclic games. SIAM Journal on Control and Optimization, 48(1):373 - 396, 2009.

[8] D. Monderer and L. S. Shapley. Potential games. Games and Economic Behavior, pages $124-143,1996$.

[9] L. Moreau and D. Aeyels. Practical stability and stabilization. IEEE Transactions on Automatic Control, pages 1554 - 1558, Aug 2000.

[10] S. Sastry. Nonlinear Systems. Springer, 1999.

[11] M. S. Stanković, K. H. Johansson, and D. M. Stipanović. Distributed seeking of Nash equilibria in mobile sensor networks. In Proc. IEEE Conf. Decision and Control, pages 5598 - 5603, 2010.

[12] M. S. Stanković and D. M. Stipanović. Discrete time extremum seeking by autonomous vehicles in a stochastic environment. In Proceedings of the 48th IEEE Conference on Decision and Control, pages 4541 - 4546, 2009.

[13] M. S. Stanković and D. M. Stipanović. Extremum seeking under stochastic noise and applications to mobile sensors. Automatica, 46:1243 - 1251, 2010.

[14] D. H. Wolpert. Theory of collective intelligence. Technical report, June 21 2003. NASA Ames Research Center, Moffett Field, CA 95033.

[15] C. Zhang, D. Arnold, N. Ghods, A. Siranosian, and M. Krstić. Source seeking with nonholonomic unicycle without position measurement and with tuning of forward velocity. Systems and Control Letters, $56: 245-252,2007$.

[16] C. Zhang, A. Siranosian, and M. Krstić. Extremum seeking for moderately unstable systems and for autonomous vehicle target tracking without position measurements. Automatica, 43:1832 - 1839, 2007.

[17] M. Zhu and S. Martinez. Distributed coverage games for mobile visual sensors (ii): Reaching the set of global optima. In Proceedings of the 48th IEEE Conference on Decision and Control, pages 175 - 180, Dec. 2009. 